\title{
Population-based study of health service deficits in US adults with depression: Does chronic disease co-morbidity and/or rural residency make a difference?
}

\author{
Andrine Lemieux, Daniel M. Saman, M. Nawal Lutfiyya* \\ Essentia Institute of Rural Health, Division of Research, Duluth, USA; 'Corresponding Author: mlutfiyya@eirh.org \\ Received 22 February 2013; revised 24 March 2013; accepted 8 April 2013 \\ Copyright (C) 2013 Andrine Lemieux et al. This is an open access article distributed under the Creative Commons Attribution License, \\ which permits unrestricted use, distribution, and reproduction in any medium, provided the original work is properly cited.
}

\section{ABSTRACT}

Introduction: Rural residents are at higher risk for a depressive disorder than their non-rural counterparts. Recent research has indicated that co-morbidities are also associated with depression. Health service deficits (HSDs) is an analytic concept that facilitates the examination of how a population uses health services relevant to their condition. A HSD is present when, over the preceding 12 months, an individual has had no health insurance, no specified health care provider, deferred medical care due to cost, or did not have a routine medical exam. Research has shown a high prevalence of HSDs in populations with individual chronic conditions. No study that we know of has examined if there is an association between the constellation of chronic conditions of depression and the comorbidities of asthma, arthritis, and diabetes, with HSDs. Methods: 2011 Behavioral Risk Factor Surveillance Survey (BRFSS) data were analyzed to identify important dimensions of the epidemiology of depression by ascertaining whether there were differences in the prevalence of health service deficits in rural versus nonrural adults with depression and at least one additional chronic disease (arthritis, asthma, or diabetes). Data analyses entailed both bivariate and multivariate techniques. All analyses were performed on weighted data. Results: Logistic regression analysis performed using the presence of at least one HSD as the dependent variable yielded that for US adults with lifetime depression those who were African American, Hispanic and other/multiracial in comparison to
Caucasian had higher odds of having at least one health service deficit. Low socioeconomic status (SES) and middle SES in comparison to high SES were also risk factors for US adults with lifetime depression having at least one HSD. Rural residency in comparison to non-rural residency also emerged as an independent risk factor (for US adults with lifetime depression having at least one HSD. Chronic disease, however, emerged as protective against US adults with lifetime depression having at least one health service deficit. Conclusions: This study demonstrated that race/ethnicity, SES, and rural residency are important predictors of health service deficits for individuals with a lifetime diagnosis of depression while having one or more chronic conditions for these same individuals was protective.

Keywords: Health Service Deficits for Depression and Other Co-Morbidities; BRFSS Surveillance Data

\section{INTRODUCTION}

Major Depressive Disorder (MDD) continues to be a significant source of disability on a global level. In 1990 MDD was ranked 15th in global disability-adjusted life years, in 2010 it moved up in rank to 11th [1]. Over that time frame the global years lived with disability due to MDD has increased 37\% (46,139 in 1990 to 63,179 in 2010) [2]. Within the United States, MDD has remained the second leading cause of years lived with disability [2]. Targeting needed resources to minimize the disability due to MDD requires a clear understanding of the patterns of disease expression and patient access and use of 
care.

Across the US there are disparities in mental health care. Rural residency increases the burden of mental health care due to disparities in practitioners and a lack of coordinated care [3]. Specific to MDD, rural residents are at higher risk for a depressive disorder and nearly three quarters of rural residents have never accessed mental health services because of a lack of resources as well as perceived and real stigma [4]. Shortages of psychiatrists and other core mental health care providers have continued to be problematic since 2004 [5]. Even when resources exist, their ability to address mental health in a comprehensive manner may be limited. For example, in the few rural communities with inpatient psychiatric services, those units more often are designed to treat geriatric populations rather than providing general adult psychiatric services [6]. When existing rural inpatient units close, patients often have to travel anywhere from 30 to 160 miles for outpatient services [6]. Stereotypes and misconceptions of mental health care provision remain high and are an additional influence on resource utilization. It has been shown that the general public, mental health providers, and rural community leaders show limited degrees of consensus on treatment issues, priorities and policies [7].

Social factors and a declining economy are additional components of medical and mental health care disparities. Living without medical insurance in the United States and having poor health significantly increases one's risk for bankruptcy, particularly when work days are lost due to illness [8]. Bankruptcy in turn has been linked to a new onset of MDD [9]. In addition to economic and geographic factors that contribute to limited care for depression, an individual's physical health, race and gender also affects receipt of care. Women are more likely to seek and receive care for depression than men and depressed women are more likely to see a medical provider than women who are not depressed [10].

Recent research has indicated that chronic disease co-morbidities are also associated with depression. When assessing the prevalence of depression in patients with osteoarthritis, Rosemann, et al. (2007) found that almost $20 \%$ of men and $19 \%$ of women had moderately severe depression as measured by the PHQ-9 [11]. Others have noted that asthma is associated with depression and that there is an increased mortality rate in depressed patients with asthma [12]. Furthermore, research has yielded that depression affects about $20 \%$ to $25 \%$ of adults with diabetes, nearly twice as many as the general medical population; and that diabetes and coexisting depression have higher all-cause mortality relative to non-depressive diabetic patients [13].

Health service deficits (HSDs) is an evolving analytic concept for use in health service-related research [14] that facilitates the examination of how a group or population might use health services relevant to their condition. A HSD is present when, over the preceding 12 months, an individual has had no health insurance, no specified health care provider, deferred medical care due to cost, or did not have a routine medical exam [14]. Using this metric, it has been demonstrated that there is a high prevalence of HSDs in populations with individual chronic conditions such as depression, arthritis and asthma [11-14].

While individual studies have examined HSDs with the distinct chronic conditions of depression, asthma, and arthritis, no studies that we know of have examined if there is an association between a constellation of chronic conditions of depression and the co-morbidities of asthma, arthritis, and diabetes, with HSDs. The purpose of this study was to ascertain the prevalence of HSDs in US adults with a self-reported health care provider diagnosis of lifetime depression with at least one additional comorbidity (asthma, arthritis or diabetes) and to explore whether rural residents experienced greater health service deficits than non-rural residents. By analyzing data from the Behavioral Risk Factor Surveillance System (BRFSS), a large national surveillance database, this study sought to fill in an important epidemiological gap regarding HSDs. Furthermore, examining data collected by a large national surveillance system such as BRFSS allows for an analysis that incorporates an array of covariates not available from clinically-based data alone. Identifying potential gaps in service for adults with lifetime depression with at least one additional chronic condition is useful for developing targeted strategies for those in need of care and not receiving it.

\section{METHODS}

2011 BRFSS data were analyzed to identify important dimensions of the epidemiology of depression by ascertaining whether there were differences in the prevalence of health service deficits in rural versus non-rural adults with self-reported lifetime depression and at least one additional chronic disease (arthritis, asthma, or diabetes). BRFSS is the largest surveillance system in the world [15]. BRFFS data are collected using a random-digit dial telephone survey targeting adults 18 through 99 years of age. These data are collected under the guidance of the Centers for Disease Control and Prevention (CDC) in collaboration with all US states and most US territories. Once collected, BRFSS data are weighted by state or territory to represent the US adult population. BRFSS data are cross-sectional and are focused on health risk factors and behaviors, health service variables, as well as chronic diseases and/or conditions. A detailed description of the survey design and sampling measures can be 
found elsewhere [15].

For this analysis, the population of interest was US adults identified as having at some point in time been diagnosed with depression by a health care providerreferred to as self-reported lifetime depression. Respondents were asked: Were you ever told (by a health care provider) that you have a depressive disorder, including depression, major depression, dysthymia, or minor depression? Inclusion criteria entailed responding yes to this question. Those responding no, don't know, or refused to answer were excluded from the analysis. The validity of such self-report methods has been confirmed in a previous study using the Structured Clinical Interview for DSM-IV (SCID-1).

All analyses were performed on weighted data as is recommended by the CDC. The weighting, calculated by the CDC, used the most recently available census data to provide a stratified representation of the nation's noninstitutionalized population.

In the analyses, presented here a number of variables were either re-coded or computed. All re-coding entailed collapsing categories and removing the responses don't know and refused. The following variables were computed: health service deficits, socioeconomic status (SES), chronic disease index, and race/ethnicity.

Health service deficits, the primary dependent variable, was computed from the response categories of a number of four different variables (health insurance status, personal healthcare provider, deferment of medical care because of cost, routine medical exam). These variables were chosen because they all impact how individuals interact with and access the health care system. The specific response categories were: did not have health insurance, did not have a healthcare provider, deferred medical care because of cost, and did not have a routine medical exam, all within the last 12 months. Having one or more of these constituted having a health service deficit.

SES, also a computed covariate, was one of the primary independent variables. According to the World Health Organization (WHO), SES is one of the strongest determinants of health [17]. While SES is a commonly used term in analyses across disciplines (e.g., sociology, social epidemiology, social psychology), many have noted that no general consensus exists about how to either define or measure the construct [18-20]. Typically SES refers to a combination of household income and other social measures such as attained educational level indexed into a single variable [18]. The most important purpose of SES is to provide a means for comparing relative position with regard to others. Almost always, SES is computed as a three-level variable (i.e., low, middle, and high) [20]. Others have noted that various measures of SES are not interchangeable and that each one assesses a different aspect of SES that reflects the intent and approach of the investigator [20]. In the current study, SES is comprised of two categorical variables: education and income. In keeping with convention, data categories from each of these individual variables were coded as low, mid-range, or high and numbered 1, 2 or 3 respectively. The variables with numbered factors or categories were then added together to create the composite variable of SES. Categories for education included: low = less than high school (coded as 1), mid-range = high school graduate (coded as 2), and high = at least some college (coded as 3). Categories for income were, low $=$ $<\$ 25,000$ (coded as 1), mid-range $=\$ 25,000-<\$ 50,000$ (coded as 2), and high $=>\$ 50,000$ (coded as 3 ). The possible range for the SES variable was 2 - 6 points. Subsequently, these points were indexed in the following manner: low $=2-3$ points, mid-range $=4-5$ points and high $=6$ points. These cut-points were purposive. For the lowest range of the index, 2 points were the floor (smallest possible point assignment), for the mid-range of the index, 4 points was the floor and for the high range of the index, 6 points was the floor. Any points below the floor for the mid-range were assigned to the lowest index category just as any points below the floor for the highest index category were assigned to the mid-range index category.

The variable chronic disease index (CDI), was constructed from the responses to three survey questions regarding whether or not the survey respondent had arthritis, lifetime asthma, and/or diabetes. These three diseases were chosen because earlier research has indicated a significant association between each of them and depression. Respondents with the specific disease or conditions were coded 1 and those without the disease or condition were coded 0 . The variables were then added together to create the composite variable of CDI. The possible range for this variable was $0-3$ and the final re-coding for this analysis was $0=$ no chronic disease and $1=$ at least one chronic disease.

The race/ethnicity variable was calculated from participant responses to two separate survey questions-one regarding race and the other regarding Latino/Hispanic ethnicity. All race/ethnicity categories were computed as mutually exclusive entities: Caucasian, African American, Hispanic, and other/multiracial. All respondents who chose white as their racial classification were coded as Caucasian; those who chose black as their racial classification were coded as African American. Respondents who chose other racial classifications including more than one race were coded as Other/multiracial. If respondents identified themselves as Hispanic or Latino, they were classified by that ethnic category regardless of any additional racial classification.

This study used the Metropolitan Statistical Area 
(MSA) variable included in BRFSS to define geographic locale. MSA was re-coded by collapsing categories into those of rural and non-rural. Rural residents were defined as persons living either within an MSA that had no city center or outside an MSA. Non-rural residents included all respondents living in a city center of an MSA, outside the city center of an MSA but inside the county containing the city center, or inside a suburban county of the MSA.

Data analyses entailed both bivariate and multivariate techniques as well some geographic information system (GIS) analysis. Bivariate analyses were performed to examine: 1) depression status (lifetime depression vs. no depression) by demographic and health-related covariates; and 2) demographic and health-related characteristics by health service deficits (at least one HSD vs. no HSD) for US adults with a health care provider diagnosis of depression. Multivariate logistic regression analysis was performed using HSDs (at least one health service deficit) as the dependent variable controlling for SES, race/ethnicity, CDI, and geographic locale. Adults with lifetime depression constituted the population examined. Additionally, the data were used to generate two different maps of the US. The first map generated displays by US state the prevalence of HSDs for US adults with depression and at least one additional chronic condition; the second displays the prevalence of lifetime depression in US adults by state.

For all statistical analyses, alpha was set at $\mathrm{p}<0.05$. Statistical Package for Social Scientists (SPSS, IBM, Chicago, IL version 19.0) was used to complete all statistical analyses performed for this study. ArcMap 10.0 was used for the GIS analysis. Human subject approval was sought and received from Essentia Health's Institutional Review Board (IRB).

\section{RESULTS}

Demographic and health related characteristics of US adults by depression status (have lifetime depression vs. no depression diagnosis) are presented in Table 1. A higher percentage of US adults with lifetime depression had at least one HSD when compared to those without a diagnosis of depression (53.2\% vs. 49.2\%). Among the HSDs, twice as many adults with lifetime depression deferred medical care because of cost when compared to those without a diagnosis (30.3\% vs. 14.0\%). A significantly higher percentage of adults with depression were also low SES (53.7\% vs. 38.8\%), rural residents $21.9 \%$ vs. $19.1 \%$ ), female (63.3\% vs. $49.0 \%$ ), not employed and not looking for employment (46.6\% vs. 33.7\%), and had at least one additional chronic disease (arthritis, asthma, and/or diabetes) (58.1\% vs. 33.9\%).

Table 2 presents the demographic and health-related characteristics of the population of US adults with lifetime depression by whether or not they had at least one health service deficit. A higher percentage of this population of adults who had at least one health service deficit were also low SES, rural, 44 years and younger, unmarried and not living with a partner, physically inactive, and had at least one chronic disease.

Table 3 displays the results of a logistic regression analysis performed using the presence of at least one HSD as the dependent variable with the covariates of race/ethnicity, SES, CDI, and geographic locale entered into the model. This analysis included only the subpopulation of US adults with lifetime depression. The results yielded that for US adults with lifetime depression those who were African American (1.087, 95\% CI = $1.083,1.090)$, Hispanic $(1.420,95 \% \mathrm{CI}=1.416,1.424)$ and other/multiracial $(1.620,95 \% \mathrm{CI}=1.614,1.625)$ in comparison to Caucasian had higher odds of having at least one health service deficit. Low SES (2.963, 95\% CI $=2.954,2.971)$ and middle SES $(1.585,95 \% \mathrm{CI}=1.581$, 1.590) in comparison to high SES were also risk factors for US adults with lifetime depression having at least one HSD. Rural residency $(1.043,95 \%$ CI $=1.041,1.045)$ in comparison to non-rural residency also emerged as an independent risk factor (albeit modestly) for US adults with lifetime depression having at least one HSD. CDI, however, emerged as protective $(0.653,95 \% \mathrm{CI}=0.651$, 0.654 ) against US adults with lifetime depression having at least one health service deficit.

By state the prevalence range for HSDs for US adults with lifetime depression and at least one chronic disease from a constellation of specific conditions was 33.50\% to 62.13\%. Figure 1 displays the prevalence data in quartiles on a map of the US. While only three states have prevalence rates in the lowest or first quartile, 15 states plus the District of Columbia constituted the second quartile, 17 states the third, and 15 states made-up the prevalence rates of the fourth quartile. With few exceptions, the majority of the states within the highest prevalence range were all from the western half of the United States. California was one exception as were Mississippi and Alabama.

Figure 2 displays by US state the quartile ranges of the prevalence of lifetime depression in US adults calculated from the 2011 BRFSS data. Prevalence ranges from a low of $10.60 \%$ to a high of $24.40 \%$. Two states constituted the lowest quartile and nine the highest. There were 20 states in the second quartile and 17 in the third.

\section{DISCUSSION}

The aim of this analysis was to determine whether chronic comorbidities and other covariates were independently associated with health service deficits among 
Table 1. Depression by demographic and health-related characteristics 2011 BRFSS data (weighted n = 232,562,614).

\begin{tabular}{|c|c|c|c|}
\hline \multirow{2}{*}{ Variable } & \multirow{2}{*}{ Factor } & \multicolumn{2}{|c|}{ Depression $^{*}$} \\
\hline & & \% Depression diagnosis & \% No depression diagnosis \\
\hline \multirow{2}{*}{ Health insurance } & Have health insurance & 80.7 & 82.3 \\
\hline & Do not have health insurance & 19.3 & 17.7 \\
\hline \multirow{2}{*}{ Health care provider } & Have health care provider & 83.0 & 77.4 \\
\hline & No health care provider & 17.0 & 22.6 \\
\hline \multirow{2}{*}{$\begin{array}{l}\text { Health care deferment because } \\
\text { of cost }\end{array}$} & Deferred health care & 30.3 & 14.0 \\
\hline & Did not defer health care & 69.7 & 86.0 \\
\hline \multirow{2}{*}{ Last routine check-up } & Within last year & 67.9 & 66.0 \\
\hline & Longer than 1 year ago & 32.1 & 34.0 \\
\hline \multirow{2}{*}{ Health service deficit ${ }^{* *}$} & At least $1 \mathrm{HSD}$ & 53.2 & 49.2 \\
\hline & No HSD & 46.8 & 50.8 \\
\hline \multirow{3}{*}{ Socioeconomic status } & Low SES & 53.7 & 38.8 \\
\hline & Mid-range SES & 37.3 & 45.5 \\
\hline & High SES & 9.0 & 15.8 \\
\hline \multirow{4}{*}{ Race/ethnicity } & Caucasian & 72.9 & 65.8 \\
\hline & African American & 8.7 & 11.8 \\
\hline & Hispanic & 12.4 & 15.3 \\
\hline & Other/multiracial & 6.0 & 7.2 \\
\hline \multirow{2}{*}{ Geographic locale } & Non-rural & 78.1 & 80.9 \\
\hline & Rural & 21.9 & 19.1 \\
\hline \multirow{2}{*}{ Respondents sex } & Male & 36.7 & 51.0 \\
\hline & Female & 63.3 & 49.0 \\
\hline \multirow{2}{*}{ Age group } & 44 years and younger & 44.9 & 48.5 \\
\hline & 45 years and older & 55.1 & 51.5 \\
\hline \multirow{2}{*}{ Marital status } & Married or living with partner & 47.4 & 57.3 \\
\hline & Unmarried not living with partner & 52.6 & 42.7 \\
\hline \multirow{3}{*}{ Employment status } & Employed & 40.6 & 58.0 \\
\hline & Unemployed & 12.8 & 8.4 \\
\hline & Not employed and not looking for employment & 46.6 & 33.7 \\
\hline \multirow{2}{*}{ Health status } & Good to excellent & 63.3 & 85.7 \\
\hline & Fair to poor & 36.7 & 14.3 \\
\hline \multirow{2}{*}{ Days physical health not good } & Fewer than 14 days & 72.2 & 91.0 \\
\hline & 14 or more days & 27.8 & 9.0 \\
\hline \multirow{2}{*}{ Days mental health not good } & Fewer than 14 days & 61.4 & 93.4 \\
\hline & 14 or more days & 38.6 & 6.6 \\
\hline \multirow{2}{*}{ Activity limitation } & Have activity limitation & 49.3 & 18.5 \\
\hline & Do not have activity limitation & 50.7 & 81.5 \\
\hline \multirow{2}{*}{ Physical activity } & Active & 43.1 & 52.7 \\
\hline & Inactive & 56.9 & 47.3 \\
\hline \multirow{2}{*}{ Arthritis } & Have arthritis & 42.5 & 21.2 \\
\hline & Do not have arthritis & 57.5 & 78.8 \\
\hline \multirow{2}{*}{ Asthma } & Asthma & 23.9 & 11.6 \\
\hline & No asthma & 76.1 & 88.4 \\
\hline \multirow{2}{*}{ Diabetes } & Diabetes & 15.3 & 8.9 \\
\hline & No diabetes & 84.7 & 91.1 \\
\hline Chronic disease index & At least 1 chronic disease & 58.1 & 33.9 \\
\hline & No chronic disease & 41.9 & 66.1 \\
\hline
\end{tabular}

*All column percentages are significantly different by z-score test for percentages; ${ }^{* *}$ Health Service Deficit is a computed variable from the four response categories of did not have health insurance, a health care provider, a routine medical checkup within the past 12 months and/or deferred needed medical care within the past 12 months because of cost; ${ }^{* * *}$ Chronic Disease Index is a computed variable comprised from the presence of at least one of three chronic conditions—arthritis, asthma and/or diabetes. 
Table 2. Demographic and health-related characteristics by health service deficits for US adults with diagnosis of depression 2011 BRFSS data (weighted $n=39,299,781$ ).

\begin{tabular}{|c|c|c|c|}
\hline \multirow{2}{*}{ Variable } & \multirow{2}{*}{ Factor } & \multicolumn{2}{|c|}{ Health service deficits ${ }^{*}$} \\
\hline & & At least 1 HSD & No HSD \\
\hline \multirow{3}{*}{ Socioeconomic status } & Low SES & 61.9 & 44.5 \\
\hline & Mid-range SES & 32.3 & 42.9 \\
\hline & High SES & 5.7 & 12.6 \\
\hline \multirow{4}{*}{ Race/ethnicity } & Caucasian & 69.0 & 77.3 \\
\hline & African American & 9.3 & 8.1 \\
\hline & Hispanic & 14.8 & 9.8 \\
\hline & Other/multiracial & 7.0 & 4.8 \\
\hline \multirow{2}{*}{ Geographic locale } & Non-rural & 77.2 & 79.0 \\
\hline & Rural & 22.8 & 21.0 \\
\hline \multirow{2}{*}{ Respondents sex } & Male & 39.0 & 34.0 \\
\hline & Female & 61.0 & 66.0 \\
\hline \multirow{2}{*}{ Age group } & 44 years and younger & 55.0 & 33.3 \\
\hline & 45 years and older & 45.0 & 66.7 \\
\hline \multirow{2}{*}{ Marital status } & Married or living with partner & 42.6 & 52.7 \\
\hline & Unmarried not living with partner & 57.4 & 47.3 \\
\hline \multirow{3}{*}{ Employment status } & Employed & 41.9 & 39.1 \\
\hline & Unemployed & 18.2 & 6.7 \\
\hline & Not employed and not looking for employment & 40.0 & 54.2 \\
\hline \multirow{2}{*}{ Health status } & Good to excellent & 61.9 & 65.0 \\
\hline & Fair to poor & 38.1 & 35.0 \\
\hline \multirow{2}{*}{ Days physical health not good } & Fewer than 14 days & 71.8 & 72.7 \\
\hline & 14 or more days & 28.2 & 27.3 \\
\hline \multirow{2}{*}{ Days mental health not good } & Fewer than 14 days & 56.6 & 66.9 \\
\hline & 14 or more days & 43.4 & 33.1 \\
\hline \multirow{2}{*}{ Activity limitation } & Have activity limitation & 49.1 & 49.4 \\
\hline & Do not have activity limitation & 50.9 & 50.6 \\
\hline \multirow{2}{*}{ Physical activity } & Active & 42.0 & 44.3 \\
\hline & Inactive & 58.0 & 55.7 \\
\hline \multirow{2}{*}{ Chronic disease index } & At least 1 chronic disease & 54.3 & 62.5 \\
\hline & No chronic disease & 45.7 & 37.5 \\
\hline
\end{tabular}

*All column percentages are significantly different by z-score test for percentages.

Table 3. Logistic regression analysis for health service deficits by race/ethnicity, SES, chronic disease index and geographic locale for US adults with depression diagnosis 2011 BRFSS data.

\begin{tabular}{llc}
\hline Covariates and Factors & & Adjusted odds ratio (95\% CI) \\
\hline \multirow{2}{*}{ Socioeconomic status } & Low SES & $2.963(2.954,2.971)$ \\
& Mid-range SES & $1.585(1.581,1.590)$ \\
& High SES & - -* $^{*}$ \\
Race/ethnicity & Caucasian & $-^{*}$ \\
& African American & $1.087(1.083,1.090)$ \\
Geographic locale & Hispanic & $1.420(1.416,1.424)$ \\
& Other/Multiracial & $1.620(1.614,1.625)$ \\
Chronic disease index & Non-rural & $--^{*}$ \\
& Rural & $1.043(1.041,1.045)$ \\
\hline
\end{tabular}

*Reference category. 


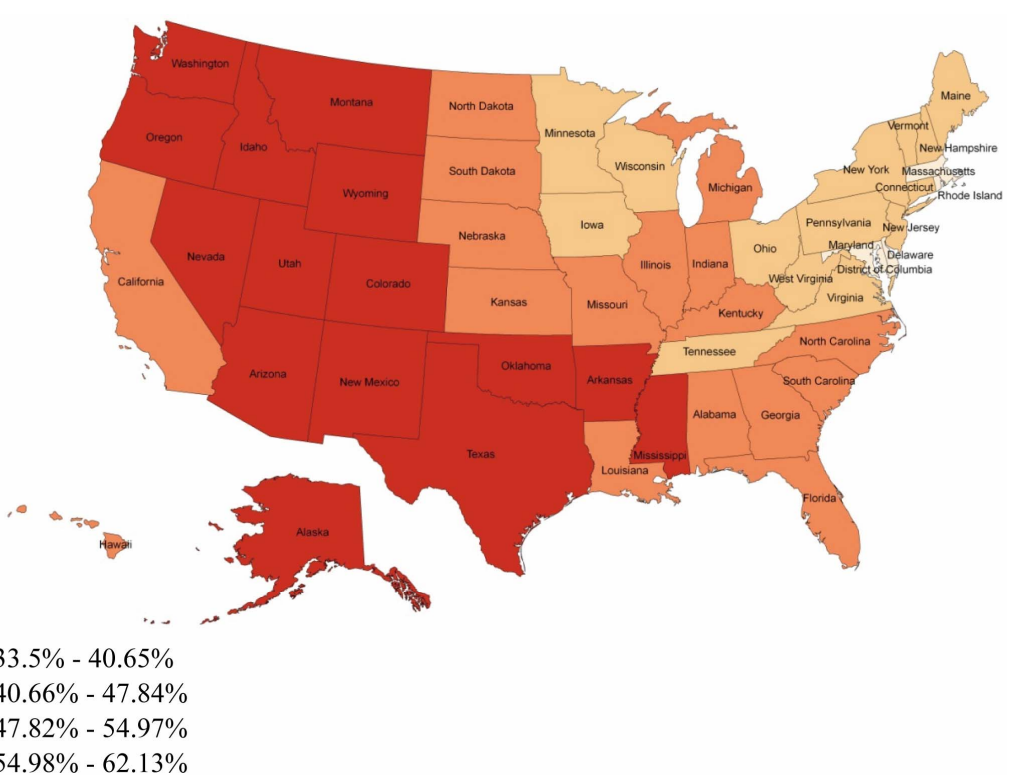

Figure 1. Prevalence of health service deficits for US adults with depression and at least one additional chronic condition 2011 BRFSS data.

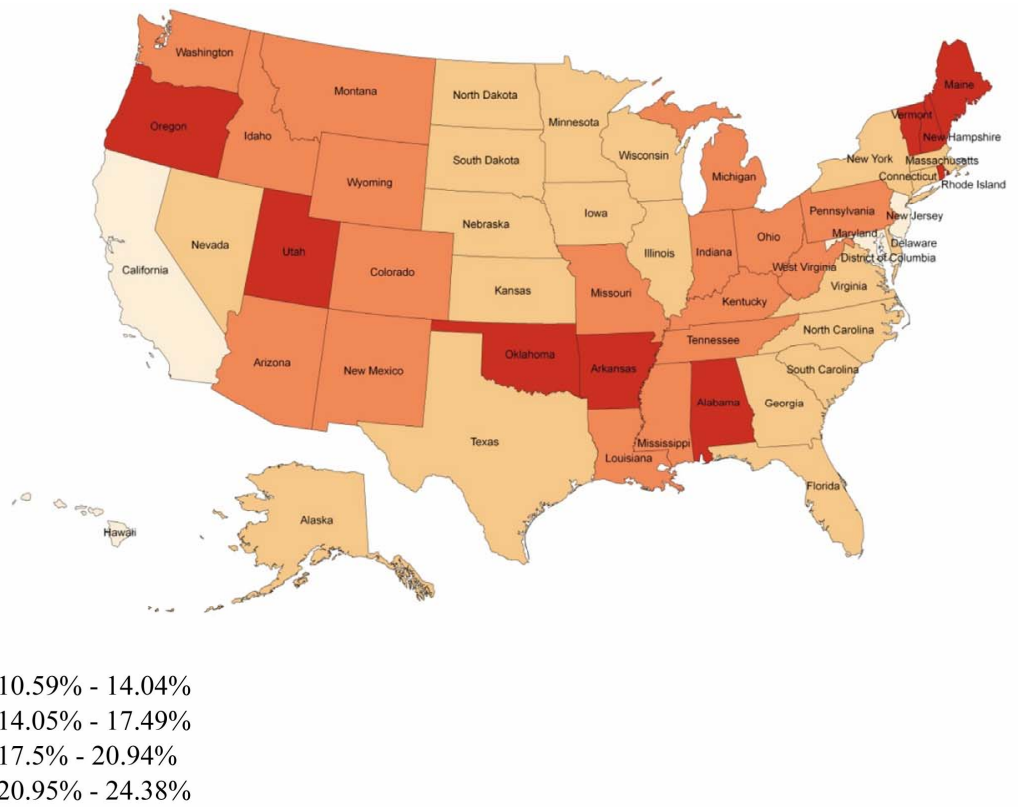

Figure 2. Prevalence of US adults with depression 2011 BRFSS data.

those with lifetime depression. Analyzing the most recent BRFSS data available (2011), three important findings emerged. First, consistent with the existing literature, this analysis yielded that a lifetime diagnosis of depression is more common among respondents with one or more chronic condition (asthma, arthritis, and diabetes). We also found there were lower odds of having a health service deficit for those with both depression and at least one chronic condition. This was true after controlling for the effects of other risk factors for HSDs such as race/ ethnicity, SES, and geographic locale. While prima facie this may seem unexpected, it is important to consider the nature of chronic conditions assessed here. All three of these conditions have been linked to a high degree of chronic pain, mortality risk, and rate of disability. In the US, seeking disability compensation inevitably requires contact with medical professionals which may partially explain this finding.

A second important finding is the further characterization of health service deficits related to race/ethnicity and SES for adults with lifetime depression. We found that low SES results in significantly increased odds of having 
health service deficits. This is not unexpected given the lack of universal health care in the US. Our results also show a gradient effect, in which not only those with low SES, but even those in the mid-range SES are at significant risk for health service deficits. Our findings also indicate that both Hispanic and Other/Multiracial respondents have much higher odds of health service deficits than African American respondents, though African American respondents continue to have significantly higher health service deficits than Caucasians. Finally, living in a rural community shows significantly increased odds of health service deficits, albeit more modestly relative to SES and race/ethnicity. This adds to the growing literature that identifies rural residency as an independent risk factor for health service deficits in general and specific to mental health [3,21].

Multiple barriers discourage patients from self-disclosing depressive symptoms to primary care physicians [22]. This analysis would suggest that health service deficits may also act as a barrier to patient access to care as well as barriers to disclosure once a patient obtains care. It may also be the case that there is an interaction between rural residency, health service deficits and willingness to disclose symptoms. For example, patients may resist disclosure to physicians when they rely on emergency department visits as a primary route of care and they know that resources in their community for addressing mental health issues are scarce or inadequate. Having resources in place for appropriate referrals is a critical component to health care provider willingness to screen for mental health issues. Our analysis suggests that this would be especially true in rural communities.

Limitations. This study is not without limitations. These limitations include three specific issues. First, the BRFSS data collection is based on telephone interviews. This may skew the collected data towards respondents with a residence and/or access to phone service. While this may be mitigated by the recent increased use of cell phone technologies, many homeless or otherwise disaffected individuals do not have access to cell phones or reliable sources of electricity to power them. To the extent that this is true, this may weaken about full understanding of health service deficits. The additional high use of answering machines and caller ID filtering of calls may have also skewed the available data. Some of these limitations were addressed by BRFSS by inclusion of US cell phone numbers in the random digit dialing approach used by BRFSS.

In addition, the data utilized here was dependent upon the accuracy of self-reported physician diagnosis of depression, arthritis, asthma and diabetes. This could have had a negative effect on estimating prevalence rates or result in misclassification. While this is undoubtedly true, at present we feel this method is superior to the use of de-identified medical records which would severely limit the amount of information available on the considerable subset of Americans with health service deficits.

Finally, the construction of health service deficits construct was restricted by BRFSS phrasing and data collection methods. It is possible that additional data could improve upon the construction of this variable. This is an important issue for further study and evaluation.

\section{CONCLUSION}

Understanding the complex interactions among mental health and medical comorbidities is an important refinement to the study of health service deficits and contributes to the epidemiological literature of depression. Depressive disorders increases mortality and morbidity related to multiple chronic health conditions, including the ones assessed here. This study supports the ongoing efforts to screen for depression in primary health care clinics and further suggests that significant segments of the population with little to no access to health care due to health service deficits continue to struggle with the combined effects of depression and chronic medical conditions.

\section{ACKNOWLEDGEMENTS}

This research was sponsored by the Rural Scientific Interest Group (Rural SIG) of the HMO Research Network (HMORN). HMORN is a consortium of health care delivery organizations with both defined patient populations and formal research capabilities. The mission of HMORN is to use collective scientific capabilities to integrate research and practice in order to improve health and health care among diverse populations. The Rural SIG seeks to facilitate collaborative research addressing the health and health care of US rural populations.

\section{REFERENCES}

[1] Murray, C.J., Vos, T., Lozano, R., Naghavi, A., Flaxman, A.D., Michaud, C., Ezzati, M., et al. (2013) Disabilityadjusted life years (DALYs) for 291 diseases and injuries in 21 regions, 1990-2010: A systematic analysis for the global burden of disease study 2010. Lancet, 380, 21972223. doi:10.1016/S0140-6736(12)61689-4

[2] Vos, T., Flaxman, A.D., Naghavi, M., Lozano, R., Michaud, C., Ezzati, M., et al. (2013) Years lived with disability (YLDs) for 1160 sequelae of 289 diseases and injuries 1990-2010: A systematic analysis for the global burden of disease study 2010. Lancet, 380, 2163-2196. doi:10.1016/S0140-6736(12)61729-2

[3] Lutfiyya, M.N., Bianco, J.A., Quinlan, S.K., Hall, C. and Waring, S.C. (2012) Mental health and mental health care in rural America: The hope of redesigned primary care. Disease-a-Month, 58, 629-638. doi:10.1016/j.disamonth.2012.08.004

[4] Bocker, E., Glasser, M., Nielsen, K. and WeidenbacherHoper, V. (2012) Rural older adults' mental health: Status 
and challenges in care delivery. Rural Remote Health, 12, 2199.

[5] National Advisory Committee on Rural Health and Human Services (2008) The 2008 Report to the Secretary: Rural Health and Human Services Issues. National Advisory Committee on Rural Health and Human Services Washington, DC.

[6] Hartley, D., Loux, S., Gale, J., Lambert, D. and Yousefian, A. (2010) Characteristics of inpatient psychiatric units in small rural hospitals. Psychiatric Services, 61, 620-623. doi:10.1176/appi.ps.61.6.620

[7] Johnsen, M.C., Morrissey, J.P., Calloway, M.O., Fried, B.J., Blank, M. and Starrett, B.E. (1997) Rural mental health leaders' perceptions of stigma and community issues. Journal of Rural Health, 13, 59-70. doi:10.1111/j.1748-0361.1997.tb00834.x

[8] Mathur, A. (2012) Health expenditures and personal bankruptcies. Health, 4, 1305-1316. doi:10.4236/health.2012.412192

[9] Ganzini, L., McFarland, B.H. and Cutler, D. (1990) Prevalence of mental disorders after catastrophic financial loss. The Journal of Nervous and Mental Disease, 178, 680-685. doi:10.1097/00005053-199011000-00002

[10] Adams, J., Sibbritt, D. and Lui, C.W. (2012) Health service use among persons with self-reported depression: A longitudinal analysis of 7164 women. Archives of Psychiatric Nursing, 26, 181-191. doi:10.1016/j.apnu.2011.10.002

[11] Rosemann, T., Laux, G. and Kuehlein, T. (2007) Osteoarthritis and functional disability: Results of a cross sectional study among primary care patients in Germany. BMC Musculoskeletal Disorders, 8, 79. doi:10.1186/1471-2474-8-79

[12] Walters, P., Schofield, P., Howard, L., Ashworth, M. and Tylee, A. (2011) The Relationship between asthma and depression in primary care patients: A historical cohort and nested case control study. PLoS One, 6, e20750. doi:10.1371/journal.pone.0020750

[13] Yavari, A. and Mashinchi, A. (2010) Diabetes and depression. Journal of Stress Physiology \& Biochemistry, 6,

\section{ABBREVIATIONS}

HSD: Health Service Deficits;

BRFSS: Behavioral Risk Factor Surveillance System;

SES: Socioeconomic Status;

MDD: Major Depressive Disorder;

PHQ-9: Patient Health Questionnaire-9;

CDC: Centers for Disease Control and Prevention;
38-43.

[14] Lutfiyya, M.N., McCullough, J.E. and Lipsky, M.S. (2011) A population-based study of health service deficits for US adults with asthma. Journal of Asthma, 48, 931-944. doi:10.3109/02770903.2011.619023

[15] Mokdad, A.H., Stroup, D.F. and Giles, W.H. (2003) Public health surveillance for behavioral risk factors in a changing environment: Recommendations from the Behavioral Risk Factor Surveillance Team. Morbidity and Mortality Weekly Report, 52, RR09.

[16] Sanchez-Villegas, A., Schlatter, J., Ortuno, F., Lahortiga, F., Pla, J., Benito, S. and Martinez-Gonzalez, M.A. (2008) Validity of a self-reported diagnosis of depression among participants in a cohort study using the Structured Clinical Interview for DSM-IV (SCID-I). BMC Psychiatry, 17, 43. doi:10.1186/1471-244X-8-43

[17] Musgrove, P., Creese, A., Preker, A., Baeza, C., Anell, A. and Prentice, T. (2000) Health systems: Improving performance. WHO, Geneva.

[18] American Psychological Association, Task Force on Socioeconomic Status (2007) Report of the APA Task Force on Socioeconomic Status. American Psychological Association, Washington DC.

[19] Department of Defense (2000) Population representation in the military services: Fiscal year 1999. Office of the Assistant Secretary of Defense, Force Management and Personnel, Washington DC.

[20] Woolfolk, A. (2007) Educational psychology. 10th Edition, Allyn and Bacon, Boston.

[21] Lutfiyya, M.N., McCullough, J.E., Haller, I.V., Waring, S.C., Bianco, J.A. and Lipsky, M.S. (2012) Rurality as a root or fundamental social determinant of health. Disease-a-Month, 58, 620-628. doi:10.1016/j.disamonth.2012.08.005

[22] Bell, R.A., Franks, P., Duberstein, P.R., Epstein, R.M., Feldman, M.D., Fernandez y Garcia, E. and Kravitz, R.L. (2011) Suffering in silence: Reasons for not disclosing depression in primary care. Annals of Family Medicine, $\mathbf{9}$, 439-446. doi:10.1370/afm.1277

WHO: World Health Organization;

CDI: Chronic Disease Index;

MSA: Metropolitan Statistical Area;

GIS: Geographic Information System;

OR: Odds Ratio;

CI: Confidence Interval. 